\title{
ZERO-HOPF BIFURCATION IN THE VOLTERRA-GAUSE SYSTEM OF PREDATOR-PREY TYPE
}

\author{
JEAN-MARC GINOUX ${ }^{1}$ AND JAUME LLIBRE ${ }^{2}$
}

\begin{abstract}
We prove that the Volterra-Gause system of predator-prey type exhibits two kinds of zero-Hopf bifurcations for convenient values of their parameters. In the first one periodic solution bifurcates from a zero-Hopf equilibrium, and in the second five periodic solutions bifurcate from another zero-Hopf equilibrium. This study is done using the averaging theory of second order.
\end{abstract}

\section{INTRODUCTION}

The very first mathematical predator-prey models have been originally proposed by Lotka [8] and Volterra [12]. In their seminal works, the interaction between prey $x$ and predator $y$ and between predator $y$ and top-predator $z$ was represented by the functional responses $x y$ and $y z$ respectively. Nevertheless, such a representation did not take into account the satiety of the predator and that of the top-predator, i.e. the saturation of the predation rate which is in this case unlimited. In the middle of the thirties Gause $[3,4]$ decided to make an "experimental verification of the mathematical theory of the struggle for existence". Then, he obtained a reasonable fit to a predation rate curve by taking the square root of $x$ (and of $y$ respectively $)^{1}$. So, he replaced the functional response $x y$ of the predator by $x^{1 / 2} y$ and that of the top-predator $y z$ can be replaced by $y^{1 / 2} z$. Thus, the Volterra-Gause model proposed by Ginoux et al. [5] is a three-dimensional model including a prey $x$, a predator $y$ and a top-predator $z$, which they named the Volterra-Gause model because it combines the original model of Volterra [12] incorporating a logisitic limitation of Verhulst [11] type on growth of the prey $x$ and a limitation of Gause $[3,4]$ type on the intensity of predation of the predator $y$ on the prey $x$ and of the top-predator $z$ on the predator $y$. In its original form the Volterra-Gause model has eight parameters but expressing its equations in a dimensionless form makes it possible to reduce this number to three. The dimensionless model is presented below.

This differential system can be written as:

$$
\begin{aligned}
& \dot{x}=a(x(1-x)-\sqrt{x} y), \\
& \dot{y}=-b y+\sqrt{x} y-\sqrt{y} z, \\
& \dot{z}=c(\sqrt{y}-d) z,
\end{aligned}
$$

2010 Mathematics Subject Classification. Primary 34C23, 34C25, 37G10.

Key words and phrases. Volterra-Gause system; predator-prey system, zero-Hopf bifurcation; periodic orbits.

${ }^{1}$ Let's note that in the beginning of the seventies, Rosenzweig [9] generalized this procedure by taking $x$ to the $g^{t h}$ power with $0<g \leqslant 1$. Thus, the predator rate reads: $x^{g} y$ for the predator and $y^{g} z$ for the top-predator. 
where $a, b, c$ and $d$ are parameters and the dot indicates derivative with respect to the time $t$. Note that the domain of definition of system (1) is

$$
D=\left\{(x, y, z) \in \mathbb{R}^{3}: x \geq 0, y \geq 0\right\} .
$$

One of the main objectives in the qualitative theory of differential systems is the study of the periodic orbits. Here we shall use the averaging theory of second order for studying the periodic orbits which bifurcate from the zero-Hopf of the Volterra-Gause system.

Here an equilibrium point with eigenvalues $\pm \omega i$ and 0 will be called a zeroHopf equilibrium, and the study of the periodic orbits which can bifurcate from this equilibrium moving the parameters of the system are the periodic orbits of a zero-Hopf bifurcation.

1.1. The zero-Hopf equilibria. First we do in $D$ the change of variables $(x, y, z, t) \mapsto$ $(X, Y, Z, s)$ given by $x=X^{2}, y=Y^{2}, z=Z$ and $t=2 s$, then system (1) becomes

$$
\begin{aligned}
& \dot{X}=a\left(X-Y^{2}-X^{3}\right), \\
& \dot{Y}=-b Y-Z+X Y, \\
& \dot{Z}=-2 c(d-Y) Z,
\end{aligned}
$$

where now the dot denotes derivative with respect to the variable $s$, and the domain of definition of this differential system is

$$
\left\{(X, Y, Z) \in \mathbb{R}^{3}: X \geq 0, Y \geq 0\right\},
$$

which we continue denoting by $D$.

Easy but tedious computations show that the equilibria of the differential system (2) in $D$ are

$$
\begin{aligned}
& E_{1}=(0,0,0), \\
& E_{2}=(1,0,0), \\
& E_{3}=\left(b, \sqrt{b-b^{3}}, 0\right) \text { when } b \in(0,1), \\
& E_{4}=\left(\frac{R}{2^{1 / 3} \cdot 3^{2 / 3}}+\frac{(2 / 3)^{1 / 3}}{R}, d, \frac{d\left(2 \cdot 3^{1 / 3}+2^{1 / 3} R^{2}\right)}{6^{2 / 3} R}-b d\right), \\
& E_{5}=\left(-\frac{(1+i \sqrt{3}) R}{2^{4 / 3} \cdot 3^{2 / 3}}-\frac{1-i \sqrt{3}}{2^{2 / 3} \cdot 3^{1 / 3} R}, d, d\left(\frac{(2 / 3)^{1 / 3}(-1+i \sqrt{3})}{2 R}-b-\frac{(\sqrt{3} i+1) R}{2^{4 / 3} \cdot 3^{2 / 3}}\right)\right),
\end{aligned}
$$

where $R=\left(\sqrt{81 d^{4}-12}-9 d^{2}\right)^{1 / 3}$, and the last two equilibria only exist when $d \in\left[0, \sqrt{2} / 3^{3 / 4}\right]$. Moreover, these last two equilibria coincide when $d=\sqrt{2} / 3^{3 / 4}$ providing the equilibrium point

$$
E_{5}=E_{6}=\left(\frac{1}{\sqrt{3}}, \frac{\sqrt{2}}{3^{3 / 4}}, \frac{\sqrt{2}(\sqrt{3}-3 b)}{3^{7 / 4}}\right) .
$$

Proposition 1. The following statements hold.

(a) The points $E_{1}$ and $E_{2}$ never are zero-Hopf equilibria.

(b) The point $E_{3}$ is a zero-Hopf equilibrium point with eigenvalues 0 and $\pm \omega i$ for the following values of the parameters

$$
(a, b, c)=\left(\frac{3 \sqrt{3}}{4} \omega^{2}, \frac{1}{\sqrt{3}}, 0\right) ;
$$


(c) The points $E_{4}$ and $E_{5}$ are only zero-Hopf equilibria with eigenvalues 0 and \pm wi when they coincide for the following values of the parameters

$$
(a, b, d)=\left(\frac{3 \sqrt{3}}{4} \omega^{2}, \frac{1}{\sqrt{3}}, \frac{\sqrt{2}}{3^{3 / 4}}\right) .
$$

Proposition 1 is proved in section 2.

We note that the equilibrium $E_{4}=E_{5}$ when $b=1 / \sqrt{3}$ coincide with the equilibrium $E_{3}$. So the following corollary is immediate:

Corollary 2. The unique zero-Hopf equilibrium point of the differential system (2) with eigenvalues 0 and $\pm \omega i$ is the equilibrium

$$
E=\left(\frac{1}{\sqrt{3}}, \frac{\sqrt{2}}{3^{3 / 4}}, 0\right)
$$

for the values of the parameters given by either (3), or (4).

Theorem 3. The equilibrium point $E$ of the Volterra-Gause system (2) exhibits a zero-Hopf bifurcation for the choice of the parameters (3) with $\omega=\sqrt{2} / 3$, when they are perturbed as follows

$$
(a, b, c)=\left(\frac{1}{2 \sqrt{3}}, \frac{1}{\sqrt{3}}+b_{2} \varepsilon^{2}, c_{2} \varepsilon^{2}\right),
$$

where $b_{2}<0$ and $c_{2}\left(\sqrt{2} \cdot 3^{1 / 4}-3 d\right) \neq 0$. Then for $\varepsilon \neq 0$ sufficiently small the periodic solution

$$
\begin{aligned}
& X(t, \varepsilon)=\frac{1}{\sqrt{3}}+\varepsilon 2^{3 / 2} \cdot 3^{-1 / 4} \sqrt{-b_{2}} \sin \frac{\sqrt{2}}{3} t+\mathcal{O}\left(\varepsilon^{2}\right) \\
& Y(t, \varepsilon)=\frac{\sqrt{2}}{3^{3 / 4}}+\varepsilon 2^{3 / 2} \sqrt{-b_{2}} \cos \frac{\sqrt{2}}{3} t+\mathcal{O}\left(\varepsilon^{2}\right) \\
& Z(t, \varepsilon)=\mathcal{O}\left(\varepsilon^{2}\right)
\end{aligned}
$$

bifurcates from $E$.

Theorem 3 is proved in section 2 .

We note that the condition $\sqrt{2} \cdot 3^{1 / 4}-3 d \neq 0$ inside Theorem 3 excludes the values of the parameters (4), because there $\sqrt{2} \cdot 3^{1 / 4}-3 d=0$.

Theorem 4. The equilibrium point $E$ of the Volterra-Gause system (2) exhibits a zero-Hopf bifurcation for the choice of the parameters (4) with $\omega=\sqrt{2} / 3$, when they are perturbed as follows

$$
(a, b, d)=\left(\frac{1}{2 \sqrt{3}}, \frac{1}{\sqrt{3}}+b_{2} \varepsilon^{2}, \frac{\sqrt{2}}{3}+d_{2} \varepsilon^{2}\right),
$$


where $b_{2}<0, d_{2}<0, c \neq 0$,

$$
\begin{aligned}
A_{1}= & \frac{6 b_{2}\left(8 \sqrt{2} c+3^{1 / 4}\right)-2\left(4 c\left(3^{1 / 4}-24 \sqrt{2} c\right)+\sqrt{6}\right) d_{2}}{\sqrt{3}-128 c^{2}}>0, \\
A_{2}= & \frac{\left(16 \cdot 3^{1 / 4} c+\sqrt{6}\right) d_{2}-12 b_{2}\left(2^{7 / 2} c+3^{1 / 4}\right)}{\sqrt{3}-128 c^{2}}>0, \\
A_{3}= & 2^{3 / 2} \cdot 3^{3 / 4} b_{2}-d_{2} \neq 0, \\
A_{4}= & -3 b_{2}-12 c d_{2}+\sqrt{2} \cdot 3^{1 / 4} d_{2} \neq 0, \text { and } \\
A_{5}= & 18\left(16 c+\sqrt{2} \cdot 3^{1 / 4}\right) b_{2}^{2}+3^{1 / 4}\left(\sqrt{6}+4 c\left(3^{1 / 4}-24 \sqrt{2} c\right)\right) d_{2}^{2} \\
& +3\left(16 c\left(24 c+\sqrt{2} \cdot 3^{1 / 4}\right)-5 \sqrt{3}\right) d_{2} b_{2} \neq 0 .
\end{aligned}
$$

(We note that there are values of the parameters $b_{2}, d_{2}$ and $c$ satisfying simultaneously the previous eight inequalities.) Then for $\varepsilon \neq 0$ sufficiently small there are 5 periodic solutions of the form

$$
\begin{aligned}
X(t, \varepsilon) & =\frac{1}{\sqrt{3}}+\varepsilon \frac{3^{3 / 4}}{\sqrt{2}}\left(w_{k}-r_{k} \frac{\sqrt{2}}{3} \sin \frac{\sqrt{2} t}{3}\right)+\mathcal{O}\left(\varepsilon^{2}\right), \\
Y(t, \varepsilon) & =\frac{\sqrt{2}}{3^{3 / 4}}+\varepsilon r_{k} \cos \frac{\sqrt{2}}{3} t+\mathcal{O}\left(\varepsilon^{2}\right), \\
Z(t, \varepsilon) & =\varepsilon w_{k}+\mathcal{O}\left(\varepsilon^{2}\right),
\end{aligned}
$$

bifurcating from $E$, where the values of $\left(r_{k}, w_{k}\right)$ for $k=1,2,3,4,5$ are given in (13).

Theorem 4 is proved in section 2 .

\section{Proof of the Results}

Proof of Proposition 1. The Jacobian matrix of the vector field associated to system (2) is

$$
M=\left(\begin{array}{ccc}
a\left(1-3 X^{2}\right) & -2 a Y & 0 \\
Y & X-b & -1 \\
0 & 2 c Z & 2 c(Y-d)
\end{array}\right)
$$

In order that the eigenvalues of the matrix $M$ at some equilibrium $E_{k}$ for $k=$ $1,2,3,4,5$ be 0 and $\pm \omega i$, the characteristic polynomial of $M$ must be $-\lambda\left(\lambda^{2}+\omega^{2}\right)$. Imposing this fact for every equilibrium $E_{k}$ for $k=1,2,3,4,5$ we obtain the results stated in the statement of the proposition.

Proof of Theorem 3. We consider system (2) for the values of the parameters given in (5). We translate the equilibrium point $E$ of this system when $\varepsilon=0$ to the origin of coordinates doing the change of variables $(X, Y, Z) \mapsto(U, V, W)$ with $X=U+b, Y=V+\sqrt{b-b^{3}}$ and $Z=W$. After we do the change of coordinates $(U, V, W) \mapsto(\bar{U}, \bar{V}, \bar{W})$ with $U=\varepsilon \bar{U}, V=\varepsilon \bar{V}$ and $W=\varepsilon \bar{W}$. Doing these two 
changes of variables system (2) becomes

$$
\dot{\bar{U}}=-\frac{\sqrt{2}}{3^{5 / 4}} \bar{V}-\frac{1}{6}\left(3 \bar{U}^{2}+\sqrt{3} \bar{V}^{2}\right) \varepsilon-\frac{1}{6} \bar{U}\left(\sqrt{3} \bar{U}^{2}+6 b_{2}\right) \varepsilon^{2}+\mathcal{O}\left(\varepsilon^{3}\right),
$$

$$
\begin{aligned}
\dot{\bar{V}} & =\frac{1}{3}\left(\sqrt{2} \cdot 3^{1 / 4} \bar{U}-3 \bar{W}\right)+\bar{U} \bar{V} \varepsilon+\mathcal{O}\left(\varepsilon^{3}\right), \\
\dot{\bar{W}} & =\frac{2}{3} c_{2}\left(\sqrt{2} \cdot 3^{1 / 4}-3 d\right) \bar{W} \varepsilon^{2}+\mathcal{O}\left(\varepsilon^{3}\right) .
\end{aligned}
$$

Doing the change of variables $(\bar{U}, \bar{V}, \bar{W}) \mapsto(u, v, w)$ with $\bar{U}=\frac{3 \sqrt{2} w-2 v}{2 \cdot 3^{1 / 4}}$, $\bar{V}=u$ and $\bar{W}=w$ the linear part of the differential system (9) is written in its real Jordan normal form, and system (9) is transformed in

$$
\begin{aligned}
\dot{u}= & -\frac{\sqrt{2}}{3} v+\varepsilon \frac{u(3 \sqrt{2} w-2 v)}{2 \cdot 3^{1 / 4}}+\mathcal{O}\left(\varepsilon^{3}\right), \\
\dot{v}= & \frac{\sqrt{2}}{3} u+\varepsilon \frac{2 u^{2}+2 v^{2}+9 w^{2}-6 \sqrt{2} v w}{4 \cdot 3^{1 / 4}}+ \\
& \varepsilon^{2}\left[c_{2}\left(2 \cdot 3^{1 / 4}-3 \sqrt{2} d\right) w+\frac{3 \sqrt{2} w-2 v}{12}\left[\frac{(3 \sqrt{2} w-2 v)^{2}}{4}+6 b_{2}\right]\right]+\mathcal{O}\left(\varepsilon^{3}\right), \\
\dot{w}= & \varepsilon^{2} \frac{2}{3} c_{2}\left(\sqrt{2} \cdot 3^{1 / 4}-3 d\right) w+\mathcal{O}\left(\varepsilon^{3}\right) .
\end{aligned}
$$

Now we change the coordinates $(u, v, w) \mapsto(r, \theta, w)$ with $u=r \cos \theta$ and $v=$ $r \cos \theta$, and taking $\theta$ as the new independent variable system (10) writes

$$
\begin{aligned}
& r^{\prime}=\varepsilon F_{11}+\varepsilon F_{21}+\mathcal{O}\left(\varepsilon^{3}\right), \\
& w^{\prime}=\varepsilon F_{12}+\varepsilon F_{22}+\mathcal{O}\left(\varepsilon^{3}\right),
\end{aligned}
$$

where the prime denotes derivative with respect to the variable $\theta$, and

$$
\begin{aligned}
F_{11}= & -\frac{3^{3 / 4} \cos (2 \theta) \sin \theta}{2 \sqrt{2}} r^{2}+\frac{3^{7 / 4}}{2} \cos (2 \theta) w r+\frac{3^{11 / 4} w^{2} \sin \theta}{4 \sqrt{2}}, \\
F_{12}= & 0, \\
F_{21}= & \frac{1}{64}\left(-16 \sqrt{2} \sin ^{4} \theta-3 \sqrt{3}(\sin (2 \theta)-4 \sin (4 \theta)+\sin (6 \theta))\right) r^{3} \\
& +\frac{9}{16}\left(4 \sin ^{3} \theta-\sqrt{6}(\cos \theta+2 \cos (3 \theta)-\cos (5 \theta))\right) w r^{2} \\
& +\frac{3}{16} \sin \theta\left(9 \sqrt{3} w^{2}(3 \cos \theta+5 \cos (3 \theta))-2 \sqrt{2}\left(9 w^{2}+4 b_{2}\right) \sin \theta\right) r \\
& +\frac{3}{16}\left(9(2 \sin \theta-3 \sqrt{6} \cos (3 \theta)) w^{2}+8\left(3 b_{2}+2^{3 / 2} \cdot 3^{1 / 4} c_{2}-6 c_{2} d\right) \sin \theta\right) w \\
& -\frac{243 \sqrt{3} \cos \theta \sin \theta w^{4}}{32 r}, \\
F_{22}= & c_{2}(2 \sqrt[4]{3}-3 \sqrt{2} d) w .
\end{aligned}
$$

Differential system (11) is written in the normal form (15) for applying the averaging theory, where using the notation of the appendix we have $t=\theta, x=(r, w)$, $T=2 \pi, n=2, F_{1}=\left(F_{11}, F_{12}\right)$ and $F_{2}=\left(F_{21}, F_{22}\right)$. Since all the assumptions of 
Theorem 5 of the appendix are satisfied we can apply it to system (11). Then the first averaged function $f_{1}(r, w)$ defined in (16) is identically zero, and the second averaged function $f_{2}(r, w)=\left(f_{21}(r, v), f_{22}(r, v)\right)$ is

$$
\begin{aligned}
& f_{21}(r, v)=-\frac{3 r\left(r^{2}+18 w^{2}+8 b_{2}\right)}{16 \sqrt{2}}, \\
& f_{22}(r, v)=c_{2}\left(2 \cdot 3^{1 / 4}-3 \sqrt{2} d\right) w .
\end{aligned}
$$

The unique zero of the second averaged function $f_{2}(r, w)$ which going back through the changes of variables are not associated to an equilibrium point of system (2) and which have $r \geq 0$ is $(r, w)=\left(2 \sqrt{-2 b_{2}}, 0\right)$, which is real because by assumptions $b_{2}<0$. Since the Jacobian of the function $f_{2}(r, w)$ at that zero is $3 b_{2} c_{2}\left(\sqrt{2} \cdot 3^{1 / 4}-3 d\right) \neq 0$ by assumptions, it follows from Theorem 5 that the differential system (11) has a periodic solution $(r(\theta, \varepsilon), w(\theta, \varepsilon))$ such that

$$
(r(0, \varepsilon), w(0, \varepsilon))=\left(2 \sqrt{-2 b_{2}}, 0\right)+\mathcal{O}(\varepsilon) .
$$

Going back through the changes of variables the periodic solution $(r(\theta, \varepsilon), w(\theta, \varepsilon))$ solution of system (11) becomes the periodic solution $(u(t, \varepsilon), v(t, \varepsilon), w(t, \varepsilon))$ of system (10) satisfying that

$$
(u(0, \varepsilon), v(0, \varepsilon), w(0, \varepsilon))=\left(2 \sqrt{-2 b_{2}} \cos (\sqrt{2} t / 3), 2 \sqrt{-2 b_{2}} \sin (\sqrt{2} t / 3), 0\right)+\mathcal{O}(\varepsilon) .
$$

And this periodic solution provides the periodic solution $(\bar{U}(t, \varepsilon), \bar{V}(t, \varepsilon), \bar{W}(t, \varepsilon))$ of system (9) such that

$(\bar{U}(0, \varepsilon), \bar{V}(0, \varepsilon), \bar{W}(0, \varepsilon))=\left(2^{3 / 2} \cdot 3^{-1 / 4} \sqrt{-b_{2}} \sin \frac{\sqrt{2} t}{3}, 2 \sqrt{-2 b_{2}} \cos \frac{\sqrt{2} t}{3}, 0\right)+\mathcal{O}(\varepsilon)$.

Finally going back first to the coordinates $(U, V, W)$ and after to $(X, Y, Z)$ we obtain the periodic solution of system (2) described in the statement of the theorem.

Now the theorem will be proved if repeating the previous computations but starting with the more general parameters

$$
(a, b, c)=\left(\frac{3 \sqrt{3}}{4} \omega^{2}+\varepsilon a_{1}+\varepsilon^{2} a_{2}, \frac{1}{\sqrt{3}}+\varepsilon b_{1}+\varepsilon^{2} b_{2}, \varepsilon c_{1}+\varepsilon^{2} c_{2}\right),
$$

we shall show that the averaged function of first order $f_{1}(r, w)=\left(f_{11}(r, v), f_{12}(r, v)\right)$ given by

$$
\begin{aligned}
& f_{11}(r, v)=\frac{r\left(\sqrt{2} \cdot 3^{3 / 4} w\left(2-9 \omega^{2}\right)-18 b_{1} \omega^{2}\right)}{8 \omega}, \\
& f_{12}(r, v)=\frac{2 c_{1}\left(\sqrt{2} \cdot 3^{1 / 4}-3 d\right) w}{3 \omega},
\end{aligned}
$$

never provide information about the periodic solutions of system (2), and consequently we need to take $f_{1}(r, w)$ identically zero doing $b_{1}=c_{1}=0, \omega=\sqrt{2} / 3$, and additionally to show that the parameters $a_{1}$ and $a_{2}$ do not play any role in the zeros of the averaged function of second order.

Indeed, the unique zeros $(r, w)$ of the function $f_{1}(r, w)$ are either $(0,0)$, or the continuums $r=0$, or $w=0$. Going back through the changes of variables the zero $(0,0)$ only produces the equilibrium point $E$ instead of a periodic solution. If we have a continuum of zeros the Jacobian determinant in these zeros of $f_{1}(r, w)$ will be zero, and the averaging theory of first order thus not provide any information 
about the periodic solutions of the system. Hence we need to take $b_{1}=c_{1}=0$ and $\omega=\sqrt{2} / 3$, for doing the first averaged function identically zero, and compute the second averaged function. Finally we note that computing the zeros of the second averaged function the parameters $a_{1}$ and $a_{2}$ do not play any role. In short we only need to study the periodic solutions for the values of the parameters given in (5), and this is what we have done at the beginning. The proof is complete.

We note that in the proof of Theorem 3 we did not provide the expressions of the corresponding differential systems (9), (10) and (11) with the values of the parameters (12) because they are very long, and really they do not provide a better understanding of the proof.

Proof of Theorem 4. We consider system (2) for the values of the parameters given in (7). Doing the same changes of variables and computations we obtain that the first averaged function is identically and the second averaged function $f_{2}(r, w)=$ $\left(f_{21}(r, v), f_{22}(r, v)\right)$ is

$$
\begin{aligned}
& f_{21}(r, v)=-\frac{3}{32} r\left(\sqrt{2} r^{2}+18\left(\sqrt{2}-4 \cdot 3^{3 / 4} c\right) w^{2}+8 \sqrt{2} b_{2}\right), \\
& f_{22}(r, v)=-\frac{3}{4} c w\left(4 \sqrt{2} d_{2}+3^{3 / 4}\left(2 r^{2}+9 w^{2}\right)\right) .
\end{aligned}
$$

When $c \neq 0$ under the assumptions of the theorem the function $f_{2}(r, w)$ has 5 zeros in $D$, namely

$$
\begin{aligned}
& \left(r_{1}, w_{1}\right)=\left(2^{3 / 2} \sqrt{-b_{2}}, 0\right) \\
& \left(r_{2}, w_{2}\right)=\left(0, \frac{2^{5 / 4}}{\sqrt{-d_{2}}}\right), \\
& \left(r_{3}, w_{3}\right)=\left(r_{2},-w_{2}\right), \\
& \left(r_{4}, w_{4}\right)=\left(\frac{2 \sqrt{A_{1}}}{3^{7 / 8}}, \frac{2 \sqrt{A_{2}}}{3^{15 / 8}}\right), \\
& \left(r_{5}, w_{5}\right)=\left(r_{4},-w_{4}\right),
\end{aligned}
$$

$A$ and $A_{2}$ are defined in the statement of the theorem. The Jacobian determinant $D_{k}$ of the function $f_{2}(r, w)$ at the zero $\left(r_{k}, w_{k}\right)$ is

$$
\begin{aligned}
D_{1} & =9 b_{2} c A_{3}, \\
D_{2}=D_{3} & =3 c d_{2} A_{4}, \\
D_{4}=D_{5} & =\frac{2 c}{\sqrt{3}-128 c^{2}} A_{5},
\end{aligned}
$$

where again $A_{3}, A_{4}$ and $A_{5}$ are defined in the statement of the theorem. Note that by assumptions $D_{k} \neq 0$ for $k=1,2,3,4,5$, and consequently by Theorem 5 , they provide 5 periodic solutions of the differential $\left(r^{\prime}, w^{\prime}\right)$ equivalent to the differential system (11) obtained with the values of the parameters given in (7). Going back these 5 periodic solutions through the changes of variables we obtain the 5 periodic solutions described in the statement of the theorem. 
The proof of the theorem will be completed if repeating the computations done in the proof of Theorem 3 but starting with the more general parameters

$$
(a, b, d)=\left(\frac{3 \sqrt{3}}{4} \omega^{2}+\varepsilon a_{1}+\varepsilon^{2} a_{2}, \frac{1}{\sqrt{3}}+\varepsilon b_{1}+\varepsilon^{2} b_{2}, \frac{\sqrt{2}}{3^{3 / 4}}+\varepsilon c_{1}+\varepsilon^{2} c_{2}\right),
$$

we shall show that the averaged function of first order $f_{1}(r, w)=\left(f_{11}(r, v), f_{12}(r, v)\right)$ given by

$$
\begin{aligned}
& f_{11}(r, v)=\frac{r\left(\sqrt{2} \cdot 3^{3 / 4} w\left(2-9 \omega^{2}\right)-18 b_{1} \omega^{2}\right)}{8 \omega}, \\
& f_{12}(r, v)=-\frac{2 c d_{1} w}{\omega},
\end{aligned}
$$

never provide information about the periodic solutions of system (2), and consequently we need to take $f_{1}(r, w)$ identically zero doing $b_{1}=d_{1}=0, \omega=\sqrt{2} / 3$, and additionally to show that the parameters $a_{1}, a_{2}$ do not play any role in the zeros of the averaged function of second order. The same arguments of the proof of Theorem 3 work here, this completes the proof of the theorem.

\section{Appendix: The AVERAging Theory of First And SECOND ORDER}

In this appendix we summarize the averaging theory of second order for finding periodic orbits. For a proof see [1] or [6]. For the general theory on averaging theory see the book [10], for another application to a zero-Hopf bifurcation see [2].

Theorem 5. Consider the differential system

$$
\dot{x}(t)=\varepsilon F_{1}(t, x)+\varepsilon^{2} F_{2}(t, x)+\varepsilon^{3} R(t, x, \varepsilon),
$$

where $F_{1}, F_{2}: \mathbb{R} \times D \rightarrow \mathbb{R}^{n}, R: \mathbb{R} \times D \times\left(-\varepsilon_{f}, \varepsilon_{f}\right) \rightarrow \mathbb{R}^{n}$ are continuous functions, $T$-periodic in the first variable, and $D$ is an open subset of $\mathbb{R}^{n}$. Assume that the following hypothesis (i) and (ii) hold.

(i) $F_{1}(t, \cdot) \in C^{1}(D)$ for all $t \in \mathbb{R}, F_{1}, F_{2}, R$ and $D_{x} F_{1}$ are locally Lipschitz with respect to $x$, and $R$ is differentiable with respect to $\varepsilon$. We define $f_{1}, f_{2}: D \rightarrow \mathbb{R}^{n}$ as

$$
\begin{aligned}
& f_{1}(z)=\int_{0}^{T} F_{1}(s, z) d s \\
& f_{2}(z)=\int_{0}^{T}\left[D_{z} F_{1}(s, z) \int_{0}^{s} F_{1}(t, z) d t+F_{2}(s, z)\right] d s .
\end{aligned}
$$

(ii) For $V \subset D$ an open and bounded set and for each $\varepsilon \in\left(-\varepsilon_{f}, \varepsilon_{f}\right) \backslash\{0\}$, there exist $a \in V$ such that $f_{1}(a)+\varepsilon f_{2}(a)=0$ and the Brouwer degree of $f_{1}+\varepsilon f_{2}$ at a is not zero.

Then for $|\varepsilon|>0$ sufficiently small, there exists a $T$-periodic solution $x(t, \varepsilon)$ of the system such that $x(0, \varepsilon) \rightarrow$ a when $\varepsilon \rightarrow 0$.

The Brouwer degree of a function $f$ at its fixed point $a$ is non-zero, if the Jacobian determinant of the function $f$ at $a$ (when it is defined) is non-zero, see for more details [7].

When the function $f_{1}$ is not identically zero, the zeros of $f_{1}+\varepsilon f_{2}$ are essentially the zeros of $f_{1}$ for $\varepsilon$ sufficiently small. Then Theorem 5 provides the averaging theory of first order. 
When the function $f_{1}$ is identically zero and $f_{2}$ is not identically zero, the zeros of $f_{1}+\varepsilon f_{2}$ are the zeros of $f_{2}$, and Theorem 5 provides the averaging theory of second order.

\section{ACKNOWLEDGMENTS}

The second author is partially supported by a MINECO grant MTM2013-40998P, an AGAUR grant number 2014SGR-568, and the grants FP7-PEOPLE-2012IRSES 318999 and 316338.

\section{REFERENCES}

[1] A. Buica And J. Llibre, Averaging methods for finding periodic orbits via Brouwer degree, Bulletin des Sciences Mathématiques 128 (2004), 7-22.

[2] C.A. Buzzi, J. Llibre and J.C. Medrado, Hopf and zero-Hopf Bifurcations on the HindmarshRose System, Nonlinear Dynamics 83 (2016), 1549-1556.

[3] G.F. Gause, The Struggle for Existence, Williams and Wilkins, Baltimore, 1934.

[4] G.F. Gause, Vérifications expérimentales de la théorie mathématique de la lutte pour la vie, Hermann, Paris, 1935 (in French).

[5] J.M. Ginoux, B. Rossetto and J.L. Jamet, Chaos In A Three-Dimensional Volterra-Gause Model Of Predator-Prey Type, International Journal of Bifurcation and Chaos 5(15) (2005), 1689-1708.

[6] J. Llibre, D.D. Novaes and M.A. Teixeira, Higher order averaging theory for finding periodic solutions via Brouwer degree, Nonlinearity 27 (2014), 563-583.

[7] N.G. Lloyd, Degree Theory, Cambridge University Press, 1978.

[8] A.J. LotkA, Elements of Physical Biology, Williams and Wilkins, Baltimore, 1925.

[9] M.L. Rosenzweig, Paradox of Enrichment: Destabilization of Exploitation Ecosystems in Ecological Time, Science, New Series, 171(3969) (1971), 385-387.

[10] J. A. Sanders F. Verhulst and J. Murdock, Averaging Methods in Nonlinear Dynamical Systems, Second edition, Applied Mathematical Sciences 59, Springer, New York, 2007.

[11] P. F. Verhulst, Notice sur la loi que suit la population dans son accroissement, Correspondances Mathemàtique et Physique 10 (1838), 113-121 (in French).

[12] V. Volterra, Variazioni e fluttuazioni del numero d'individui in specie animali conviventi, Memoria della Reale Accademia Nazionale dei Lincei III (6) (1926), 31-113 (in Italian).

1 Laboratoire LSIS, CNRS, UMR 7296, Université de Toulon, BP 20132, F-83957 La Garde CEDEX, France

E-mail address: ginoux@univ-tln.fr

2 Departament de Matemàtiques, Universitat Autònoma de Barcelona, 08193 Bellaterra, Barcelona, Catalonia, Spain

E-mail address: jllibre@mat.uab.cat 